Commun.Fac.Sci.Univ.Ank.Series A 1

Volume 58, Number 1, Pages 39-48 (2009)

ISSN $1303-5991$

\title{
INTERVAL OSCILLATION CRITERIA FOR SECOND-ORDER DELAY AND ADVANCED DIFFERENCE EQUATIONS
}

\author{
A. FEZA GÜVENILIR
}

ABstRACT. Interval oscillation criteria are established for second-order difference equations in the form

$$
\Delta(k(n) \Delta x(n))+p(n) x(g(n))+q(n)|x(g(n))|^{\gamma-1} x(g(n))=e(n), \quad\left(E_{\gamma}\right)
$$
where $n \geq n_{0}, n_{0} \in \mathbb{N}=\{0,1, \ldots\}, \quad \gamma>1 ; k, p, q, e$ and $g$ are sequences of real numbers; $k(n)>0$ is nondecreasing; $g(n)$ is nondecreasing, $\lim _{n \rightarrow \infty} g(n)=\infty$. Several oscillation criteria are given for equation $\left(E_{\gamma}\right)$ considered as to separate delay and advanced difference equations when $g(n)<n$ and $g(n)>n$ respectively. Illustrative examples are included.

\section{Introduction}

We consider second-order difference equations of the form,

$$
\Delta(k(n) \Delta x(n))+p(n) x(g(n))+q(n)|x(g(n))|^{\gamma-1} x(g(n))=e(n) \quad\left(E_{\gamma}\right)
$$

where $n \geq n_{0}, n_{0} \in \mathbb{N}=\{0,1, \ldots\}, \gamma>1 ; k, p, q, e$ and $g$ are sequences of real numbers; $k(n)>0$ is nondecreasing; $g(n)$ is nondecreasing, $\lim _{n \rightarrow \infty} g(n)=\infty$. $\Delta$ is the forward difference operator defined by $\Delta x(n)=x(n+1)-x(n)$. As is customary, we assume that solutions of $\left(E_{\gamma}\right)$ exist on some set $\left\{n_{0}, n_{0}+1, \ldots\right\}$. For the theory of existence of solutions of such equations, we refer [1]. A nontrivial solution $\{x(n)\}$ of $\left(E_{\gamma}\right)$ is called oscillatory if for any given $\tilde{n}_{0} \geq n_{0}$ there exists an integer $n_{1} \geq \tilde{n}_{0}$ such that $x\left(n_{1}\right) x\left(n_{1}+1\right) \leq 0$, otherwise it is called nonoscillatory. The equation will be called oscillatory if every solution is oscillatory. Taking $g(n)$ as $\tau(n)$ with $\tau(n)<n$ and $\lim _{n \rightarrow \infty} \tau(n)=\infty, \gamma=\alpha$, equation $\left(E_{\gamma}\right)$ is considered as a delay difference equation

$$
\Delta(k(n) \Delta x(n))+p_{1}(n) x(\tau(n))+q_{1}(n)|x(\tau(n))|^{\alpha-1} x(\tau(n))=e(n) \quad\left(E_{D}\right)
$$

Received by the editors April 16, 2009, Accepted: June. 16, 2009.

2000 Mathematics Subject Classification. 34K11, 34C10.

Key words and phrases. Interval oscillation, Second-order, Delay argument, Advanced argument, Oscillatory. 
or taking $g(n)$ as $\sigma(n)$ with $\sigma(n)>n$ and $\gamma=\beta$,equation $\left(E_{\gamma}\right)$ is considered as an advanced difference equation

$$
\Delta(k(n) \Delta x(n))+p_{2}(n) x(\sigma(n))+q_{2}(n)|x(\sigma(n))|^{\beta-1} x(\sigma(n))=e(n) . \quad\left(E_{A}\right)
$$

In literature, there isn't enough work dealing with the oscillation of difference equations $\left(E_{D}\right)$ and $\left(E_{A}\right)$. Equation $\left(E_{\gamma}\right)$, when $k(n) \equiv 1, p(n) \equiv 0$ or $q(n) \equiv 0$ and $g(n)=n, n+1, n-\tau$ has been studied by many authors, see $[6,7,12,13,15]$ and the references cited therein.

Using Riccatti tecnique, Saker[9] obtained some oscillation criteria for forced Emden-Fowler superlinear difference equation of the form

$$
\Delta^{2} x(n)+q(n) x^{\gamma}(n+1)=e(n)
$$

when $q(n)$ and $e(n)$ are sequences of positive real numbers.

Zhang and Chen [14] established some oscillation criteria

$$
\Delta^{2} x(n)+q(n) f(x(n+1))=0
$$

when $f$ is nondecreasing and $u f(u)>0$ for $u \neq 0$.

The first result concerning the interval oscillation of $(E \gamma)$ when $g(n)=n+1$, $q(n) \equiv 0, e(n) \equiv 0$ has been studied by Kong and Zettl [7]. They have applied the telescoping principle for equation of the form

$$
\Delta(k(n) \Delta x(n))+p(n) x(n+1)=0 .
$$

Recently, Güvenilir and Zafer [4] has presented some sufficient conditions about oscillation of second-order differential equation

$$
\left(k(t) x^{\prime}(t)\right)^{\prime}+p(t)|x(\tau(t))|^{\alpha-1} x(\tau(t))+q(t)|x(\sigma(t))|^{\beta-1} x(\sigma(t))=e(t) .
$$

where $n \geq 0$. Later, in [2] Anderson generalized the results of Güvenilir and Zafer [4] to the dynamic equation

$$
\left(k x^{\Delta}\right)^{\Delta}(t)+p(t)|x(\tau(t))|^{\alpha-1} x(\tau(t))+q(t)|x(\sigma(t))|^{\beta-1} x(\sigma(t))=e(t)
$$

where $n \geq 0$ for arbitrary time scales.

In this work, our purpose is to derive interval oscillation criteria as discrete analogues of the ones contained [3]. The difference between $\left(E_{\gamma}\right)$ and (1.2) is the appearence of both linear and nonlinear terms. Therefore, the results in [2] fails to apply for $\left(E_{\gamma}\right)$.

For our purpose, we denote

$$
D\left(a_{k}, b_{k}\right)=\left\{u: u\left(a_{k}\right)=u\left(b_{k}\right)=0, k=1,2, u(n) \not \equiv 0, n \in \mathbb{N}\left(a_{k}, b_{k}\right)\right\},
$$

where $\mathbb{N}\left(a_{k}, b_{k}\right)=\left\{a_{k}, a_{k}+1, \ldots, b_{k}\right\}$. As in [4], we define

$$
P_{*}(n)=*(*-1)^{1 / *-1} q(n)^{1 / *}|e(n)|^{1-1 / *} .
$$




\section{Delay Difference Equations}

Suppose that for any given $N \geq 0$ there exist $a_{1}, a_{2}, b_{1}, b_{2} \geq N$ such that $a_{1}<b_{1}$, $a_{2}<b_{2}$ and

$$
p_{1}(n) \geq 0, q_{1}(n) \geq 0 \text { for } n \in \mathbb{N}\left(\tau\left(a_{1}\right), b_{1}\right) \cup \mathbb{N}\left(\tau\left(a_{2}\right), b_{2}\right) .
$$

Let $e(n)$ satisfies

$$
\begin{aligned}
& e(n) \leq 0, \text { for } n \in \mathbb{N}\left(\tau\left(a_{1}\right), b_{1}\right) \\
& e(n) \geq 0, \text { for } n \in \mathbb{N}\left(\tau\left(a_{2}\right), b_{2}\right) .
\end{aligned}
$$

Theorem 2.1. Suppose that (2.1) and (2.2) hold. If there exist an $H_{1} \in$ $D\left(a_{i}, b_{i}\right), i=1,2$, such that

$$
\sum_{n=a_{i}}^{b_{i}-1}\left[H_{1}^{2}(n+1)\left(p_{1}(n)+P_{\alpha}(n)\right) \frac{\tau(n)-\tau\left(a_{i}\right)}{n+1-\tau\left(a_{i}\right)}-\left(\Delta H_{1}(n)\right)^{2} k(n)\right] \geq 0,
$$

for $i=1,2$, then $\left(E_{D}\right)$ is oscillatory.

Proof. To get a contradiction, let us suppose that $x(n)$ is a nonoscillatory solution of equation $\left(E_{D}\right)$. First, assume $x(n)>0, x(\tau(n))>0$ for all $n \geq n_{1}$ for some $n_{1}>0$.

We may say

$$
F(x)=A x^{\mu}-\mu(\mu-1)^{1 / \mu-1} A^{1 / \mu} B^{1-1 / \mu} x+B \geq 0 \text { for } x \in[0, \infty)
$$

where $A, B$ are nonnegative constants and $\mu>1,[10]$.

If we choose $A=q_{1}(t), B=-e(n)$ and $\mu=\alpha$ in (2.4), we have

$$
q_{1}(t) x^{\alpha}(\tau(n))-e(n) \geq \alpha(\alpha-1)^{1 / \alpha-1} q_{1}(n)^{\frac{1}{\alpha}}|e(n)|^{1-\frac{1}{\alpha}} x(\tau(n)) .
$$

for $n \in \mathbb{N}\left(\tau\left(a_{1}\right), b_{1}\right)$

See also $[8,10]$.

Define

$$
w(n)=-\frac{k(n) \Delta x(n)}{x(n)}, n \geq n_{1}, n_{1}>0 .
$$

In view of $\left(E_{D}\right)$, we see that

$$
\begin{aligned}
& \Delta w(n)=\frac{x(n)}{k(n) x(n+1)} w^{2}(n)+p_{1}(n) \frac{x(\tau(n))}{x(n+1)} \\
& +\left[q_{1}(n) x^{\alpha}(\tau(n))-e(n)\right] \frac{1}{x(n+1)} .
\end{aligned}
$$

Using (2.1) and (2.5), we see from (2.7) that

$$
\Delta w(n) \geq \frac{x(n)}{k(n) x(n+1)} w^{2}(n)+\left[p_{1}(n)+P_{\alpha}(n)\right] \frac{x(\tau(n))}{x(n+1)}, n \in \mathbb{N}\left(\tau\left(a_{1}\right), b_{1}\right) .
$$

Moreover

$$
x(n+1)=x(n)+\Delta x(n),
$$




$$
\frac{x(n+1)}{x(n)}=1+\frac{\Delta x(n)}{x(n)}
$$

and then

$$
\frac{x(n)}{k(n) x(n+1)}=\frac{1}{k(n)-w(n)} .
$$

Therefore

$$
\Delta w(n) \geq \frac{1}{k(n)-w(n)} w^{2}(n)+\left[p_{1}(n)+P_{\alpha}(n)\right] \frac{x(\tau(n))}{x(n+1)}, n \in \mathbb{N}\left(\tau\left(a_{1}\right), b_{1}\right) .
$$

Now by the Mean Value Theorem in [1]

$$
x(n)-x\left(\tau\left(a_{1}\right)\right) \geq \frac{k(\xi) \Delta x(\xi)}{k(\xi)}\left(n-\tau\left(a_{1}\right)\right)
$$

for some $\xi \in \mathbb{N}\left(\tau\left(a_{1}\right), n\right)$. From which, for any $n \in \mathbb{N}\left(a_{1}, b_{1}\right)$,we have

$$
x(n) \geq \Delta x(n)\left(n-\tau\left(a_{1}\right)\right), n \in \mathbb{N}\left(a_{1}, b_{1}\right)
$$

and hence,

$$
\frac{\Delta x(n)}{x(n)} \leq \frac{1}{n-\tau\left(a_{1}\right)}, n \in \mathbb{N}\left(a_{1}, b_{1}\right)
$$

Moreover, following the arguments in [2], since

$$
x(m)-\Delta x(m)\left(m-\tau\left(a_{1}\right)\right) \geq 0, m \in \mathbb{N}(\tau(n), n+1), n \in \mathbb{N}\left(a_{1}, b_{1}\right)
$$

we have

$$
\frac{x(m)-\Delta x(m)\left(m-\tau\left(a_{1}\right)\right)}{x(m) x(m+1)} \geq 0 .
$$

Therefore,

$$
\Delta\left(\frac{m-\tau\left(a_{1}\right)}{x(m)}\right) \geq 0
$$

It follows that

$$
\sum_{m=\tau(n)}^{n} \Delta\left(\frac{m-\tau\left(a_{1}\right)}{x(m)}\right)=\frac{n+1-\tau\left(a_{1}\right)}{x(n+1)}-\frac{\tau(n)-\tau\left(a_{1}\right)}{x(\tau(n))},
$$

in other words

$$
\frac{x(\tau(n))}{x(n+1)} \geq \frac{\tau(n)-\tau\left(a_{1}\right)}{n+1-\tau\left(a_{1}\right)}, n \in \mathbb{N}\left(a_{1}, b_{1}\right)
$$


In view of (2.9), it follows from (2.8) that

$$
\Delta w(n) \geq \frac{1}{k(n)-w(n)} w^{2}(n)+\left[p_{1}(n)+P_{\alpha}(n)\right] \frac{\tau(n)-\tau\left(a_{1}\right)}{n+1-\tau\left(a_{1}\right)}, n \in \mathbb{N}\left(\tau\left(a_{1}\right), b_{1}\right) .
$$

Let $H_{1} \in D\left(a_{1}, b_{1}\right)$ be given as in the hypothesis. Multiplying $H_{1}^{2}(n+1)$ through (2.10) we find

$$
\begin{aligned}
\Delta w(n) H_{1}^{2}(n+1) & \geq \frac{1}{k(n)-w(n)} w^{2}(n) H_{1}^{2}(n+1) \\
& +\left[p_{1}(n)+P_{\alpha}(n)\right] \frac{\tau(n)-\tau\left(a_{1}\right)}{n+1-\tau\left(a_{1}\right)} H_{1}^{2}(n+1)
\end{aligned}
$$

for $n \in \mathbb{N}\left(\tau\left(a_{1}\right), b_{1}\right)$. Since

$$
\begin{aligned}
& \Delta\left(H_{1}^{2}(n) w(n)\right)=H_{1}^{2}(n+1) \Delta w(n)+w(n) \Delta H_{1}^{2}(n) \\
& \begin{aligned}
\Delta\left(H_{1}^{2}(n)\right) & =\Delta\left(H_{1}(n) H_{1}(n)\right) \\
& =H_{1}(n+1) \Delta H_{1}(n)+H_{1}(n) \Delta H_{1}(n) \\
& =\Delta H_{1}(n)\left(H_{1}(n+1)+H_{1}(n)\right)
\end{aligned}
\end{aligned}
$$

and

$$
\Delta\left(H_{1}^{2}(n)\right)=\Delta H_{1}(n)\left[2 H_{1}(n+1)-\Delta H_{1}(n)\right]
$$

then taking the sum from $a_{1}$ to $\left(b_{1}-1\right)$ we obtain

$$
\begin{aligned}
& \sum_{n=a_{1}}^{b_{1}-1}\left\{\left[p_{1}(n)+P_{\alpha}(n)\right] \frac{\tau(n)-\tau\left(a_{1}\right)}{n+1-\tau\left(a_{1}\right)} H_{1}^{2}(n+1)-k(n)\left(\Delta H_{1}(n)\right)^{2}\right\} \\
\leq & -\Delta H_{1}^{2} w\left(a_{1}\right)-\sum_{n=a_{1}}^{b_{1}-1}\left[\frac{w(n) H_{1}(n+1)}{\sqrt{k(n)-w(n)}}+\sqrt{k(n)-w(n)} \Delta H_{1}(n)\right]^{2} . \\
& \sum_{n=a_{1}}^{b_{1}-1}\left\{\left[p_{1}(n)+P_{\alpha}(n)\right] \frac{\tau(n)-\tau\left(a_{1}\right)}{n+1-\tau\left(a_{1}\right)} H_{1}^{2}(n+1)-k(n)\left(\Delta H_{1}(n)\right)^{2}\right\} \\
\leq & -\sum_{n=a_{1}}^{b_{1}-1}\left[\frac{w(n) H_{1}(n+1)}{\sqrt{k(n)-w(n)}}+\sqrt{k(n)-w(n)} \Delta H_{1}(n)\right]^{2}<0 .
\end{aligned}
$$

Note that

$$
\sum_{n=a_{1}}^{b_{1}-1}\left[\frac{w(n) H_{1}(n+1)}{\sqrt{k(n)-w(n)}}+\sqrt{k(n)-w(n)} \Delta H_{1}(n)\right]^{2}=0
$$


is possible only if

$$
\frac{w(n) H_{1}(n+1)}{\sqrt{k(n)-w(n)}}+\sqrt{k(n)-w(n)} \Delta H_{1}(n)=0 .
$$

Therefore

$$
\begin{aligned}
& -\frac{w(n) H_{1}(n+1)}{\sqrt{k(n)-w(n)}}=\sqrt{k(n)-w(n)} \Delta H_{1}(n) \\
& -w(n) H_{1}(n+1)=(k(n)-w(n)) \Delta H_{1}(n)
\end{aligned}
$$

and then

$$
\begin{gathered}
\frac{k(n) \Delta x(n)}{x(n)} H_{1}(n+1)=\frac{k(n) x(n+1)}{x(n)} \Delta H_{1}(n) \\
\Delta x(n) H_{1}(n+1)=x(n+1) \Delta H_{1}(n) .
\end{gathered}
$$

Hence

which implies

$$
\Delta\left(\frac{H_{1}(n)}{x(n)}\right)=0
$$

$$
H_{1}(n)=c x(n),
$$

where $c$ is a constant. This, however, contradicts the positivity of $x(n)$. Now (2.11) contradicts (2.3). Thus, the proof is complete, when $x(n)$ is eventually positive. The proof can be accomplished similarly by working with $\mathbb{N}\left(a_{2}, b_{2}\right)$ instead of $\mathbb{N}\left(a_{1}, b_{1}\right)$ when $x(n)$ is eventually negative.

Example 2.1. Consider the forced delay difference equation,

$$
\Delta^{2} x(n)+m_{1} \sin \left(\frac{\pi n}{60}\right) x(n-2)+m_{2} \cos \left(\frac{\pi n}{60}\right) x^{3}(n-2)=\cos \left(\frac{\pi n}{10}\right)
$$

where $m_{1}, m_{2}>0$. Let

$$
\begin{aligned}
& a_{1}=8+120 k, \quad b_{1}=11+120 k, \\
& a_{2}=17+120 k, \quad b_{2}=20+120 k
\end{aligned}
$$

for any nonnegative integer $k$ and let $H_{1}(n)=\sin \left(\pi \frac{(n+1)}{3}\right)$. It is easy to check that (2.1) is satisfied, namely

$p_{1}(n)=m_{1} \sin \left(\frac{\pi n}{60}\right) \geq 0$, for $n \in \mathbb{N}(6+120 k, 11+120 k) \cup(15+120 k, 20+120 k)$.

$q_{1}(n)=m_{2} \cos \left(\frac{\pi n}{60}\right) \geq 0$, for $n \in \mathbb{N}(6+120 k, 11+120 k) \cup(15+120 k, 20+120 k)$.

and 


$$
\begin{gathered}
e(n)=\cos \left(\frac{\pi n}{10}\right) \leq 0, \text { for } n \in \mathbb{N}(6+120 k, 11+120 k) \\
e(n)=\cos \left(\frac{\pi n}{10}\right) \geq 0, \text { for } n \in \mathbb{N}(15+120 k, 20+120 k)
\end{gathered}
$$

where $\tau(n)=n-2$.

By Theorem 2.1, the equation (2.12) is oscillatory when $m_{1}=1, m_{2}>79$; when $m_{2}=1, m_{1}>14$.

\section{Advanced Difference Equations}

\section{Consider}

$$
\Delta(k(n) \Delta x(n))+p_{2}(n) x(\sigma(n))+q_{2}(n)|x(\sigma(n))|^{\beta-1} x(\sigma(n))=e(n) . \quad\left(E_{A}\right)
$$

where $n \geq n_{0}, n_{0} \in \mathbb{N}=\{0,1, \ldots\}, \beta>1, k, p_{2}, q_{2}, e$ and $\sigma$ are sequences of real numbers, $k(n)>0$ is nondecreasing; $\sigma(n)>n, \sigma$ is nondecreasing. Suppose that for any given $N \geq 0$ there exist $c_{1}, c_{2}, d_{1}, d_{2} \geq N$ such that $c_{1}<d_{1}, c_{2}<d_{2}$ and

$$
p_{2}(n) \geq 0, q_{2}(n) \geq 0, \text { for } n \in \mathbb{N}\left(c_{1}, \sigma\left(d_{1}\right)\right) \cup \mathbb{N}\left(c_{2}, \sigma\left(d_{2}\right)\right) .
$$

Let $e(n)$ satisfies

$$
\begin{gathered}
e(n) \leq 0, \text { for } n \in \mathbb{N}\left(c_{1}, \sigma\left(d_{1}\right)\right) \\
e(n) \geq 0, \text { for } n \in \mathbb{N}\left(c_{2}, \sigma\left(d_{2}\right)\right) .
\end{gathered}
$$

Now, we can give the following .

Theorem 3.1. Suppose that (3.1) and (3.2) hold. If there exist an $H_{2} \in$ $D\left(c_{i}, d_{i}\right)$ such that

$$
\sum_{n=c_{i}}^{d_{i}-1}\left[H_{2}^{2}(n+1)\left(p_{2}(n)+P_{\beta}(n)\right) \frac{\sigma\left(d_{i}\right)-\sigma(n)}{\sigma\left(d_{i}\right)-(n+1)}-\left(\Delta H_{2}(n)\right)^{2} k(n)\right] \geq 0
$$

for $i=1,2$, then $\left(E_{A}\right)$ is oscillatory.

Proof. To arrive at a contradiction, let us suppose that $x(n)$ is a nonoscillatory solution of equation $\left(E_{A}\right)$. First, assume $x(n), x(\sigma(n))$ are positive for all $n \geq$ $n_{1}$ for some $n_{1}>0$.

Considering (2.6), in view of $\left(E_{A}\right)$, we see that

$$
\begin{aligned}
\Delta w(n)= & \frac{x(n)}{k(n) x(n+1)} w^{2}(n)+p_{2}(n) \frac{x(\sigma(n))}{x(n+1)} \\
& +\left[q_{2}(n) x^{\beta}(\sigma(n))-e(n)\right] \frac{1}{x(n+1)} .
\end{aligned}
$$

In (2.5) instead of $\tau(n), \alpha$ and $q_{1}$ we take $\sigma(n), \beta$ and $q_{2}$ respectively, we get 


$$
\Delta w(n) \geq \frac{x(n)}{k(n) w(n+1)} w^{2}(n)+\left[p_{2}(n)+P_{\beta}(n)\right] \frac{x(\sigma(n))}{x(n+1)}, n \in \mathbb{N}\left(c_{1}, \sigma\left(d_{1}\right)\right) .
$$

By the same steps in Theorem 2.1, we obtain

$$
\Delta w(n) \geq \frac{1}{k(n)-w(n)} w^{2}(n)+\left[p_{2}(n)+P_{\beta}(n)\right] \frac{x(\sigma(n))}{x(n+1)}, n \in \mathbb{N}\left(c_{1}, \sigma\left(d_{1}\right)\right) .
$$

Note that $\Delta(k(n) \Delta x(n)) \leq 0$ on $\left[c_{1}, \sigma\left(d_{1}\right)\right]$. In a similar manner as in the proof of Theorem (2.1) we get

$$
\frac{x(\sigma(n))}{x(n+1)} \geq \frac{\sigma\left(d_{1}\right)-\sigma(n)}{\sigma\left(d_{1}\right)-(n+1)}, n \in \mathbb{N}\left(c_{1}, \sigma\left(d_{1}\right)\right) .
$$

Applying inequality (3.5) to (3.4), we obtain

$\Delta w(n) \geq \frac{1}{k(n)-w(n)} w^{2}(n)+\left[p_{2}(n)+P_{\beta}(n)\right] \frac{\sigma\left(d_{1}\right)-\sigma(n)}{\sigma\left(d_{1}\right)-(n+1)}, n \in \mathbb{N}\left(c_{1}, \sigma\left(d_{1}\right)\right)$.

Using the same steps in the proof of Theorem (2.1) we get

$$
\begin{aligned}
& \sum_{n=c_{1}}^{d_{1}-1}\left\{\left[p_{2}(n)+P_{\beta}(n)\right] \frac{\sigma\left(d_{1}\right)-\sigma(n)}{\sigma\left(d_{1}\right)-(n+1)} H_{2}^{2}(n+1)-k(n)\left(\Delta H_{2}(n)\right)^{2}\right\} \\
\leq & -\sum_{n=c_{1}}^{d_{1}-1}\left[\frac{w(n) H_{2}(n+1)}{\sqrt{k(n)-w(n)}}+\sqrt{k(n)-w(n)} \Delta H_{2}(n)\right]^{2}<0
\end{aligned}
$$

(3.6) contradicts (3.3). Thus the proof is complete, when $x(n)$ is eventually positive. The proof can be accomplished similarly by working with $\mathbb{N}\left(c_{2}, d_{2}\right)$ instead of $\mathbb{N}\left(c_{1}, d_{1}\right)$ when $x(n)$ is eventually negative.

Example 3.1. Consider the advanced difference equation,

$$
\Delta^{2} x(n)+m_{1} \sin \left(\frac{\pi n}{60}\right) x(n+2)+m_{2} \cos \left(\frac{\pi n}{60}\right) x^{3}(n+2)=\cos \left(\frac{\pi n}{10}\right)
$$

where $m_{1}, m_{2} \geq 0$. Let

$$
\begin{aligned}
& c_{1}=6+120 k, d_{1}=9+120 k, \\
& c_{2}=15+120 k, d_{2}=18+120 k
\end{aligned}
$$

for any nonnegative integer $k$ and let $H_{2}(n)=\sin \left(\frac{n \pi}{3}\right)$. It is easy to check that (3.1) is satisfied, namely 
$p_{2}(n)=m_{1} \sin \left(\frac{\pi n}{60}\right) \geq 0$, for $n \in \mathbb{N}(6+120 k, 11+120 k) \cup(15+120 k, 20+120 k)$.

$q_{2}(n)=m_{2} \cos \left(\frac{\pi n}{60}\right) \geq 0$, for $n \in \mathbb{N}(6+120 k, 11+120 k) \cup(15+120 k, 20+120 k)$.

and

$$
\begin{gathered}
e(n)=\cos \left(\frac{\pi n}{10}\right) \leq 0, \text { for } n \in \mathbb{N}(6+120 k, 11+120 k) \\
e(n)=\cos \left(\frac{\pi n}{10}\right) \geq 0, \text { for } n \in \mathbb{N}(15+120 k, 20+120 k)
\end{gathered}
$$

where $\sigma(n)=n+2$.

By Theorem 3.1, the equation (3.7) is oscillatory when $m_{1}=1, m_{2}>10$; when $m_{2}=1, m_{1}>1$.

\section{Delay and Advanced Difference Equations}

We obtain the delay and advanced difference equations as follows:

$$
\begin{gathered}
\Delta(k(n) \Delta x(n))+p_{1}(n) x(\tau(n))+q_{1}(n)|x(\tau(n))|^{\alpha-1} x(\tau(n)) \\
+p_{2}(n) x(\sigma(n))+q_{2}(n)|x(\sigma(n))|^{\beta-1} x(\sigma(n))=e(n),
\end{gathered}
$$

where $n \geq n_{0}, n_{0} \in \mathbb{N}=\{0,1, \ldots\}, \beta>1, k, p_{1}, p_{2}, q_{1}, q_{2}, e, \tau$ and $\sigma$ are sequences of real numbers, $k(n)>0$ is nondecreasing; $\tau(n)<n, \sigma(n)>n, \tau$ and $\sigma$ are nondecreasing and $\lim _{\mathbf{t} \rightarrow \infty} \tau(t)=\infty$.

Suppose that for any given $N \geq 0$ there exist $a_{1}, a_{2}, b_{1}, b_{2}, c_{1}, c_{2}, d_{1}, d_{2} \geq N$ such that $a_{1}<b_{1}, a_{2}<b_{2}$ and $c_{1}<d_{1}, c_{2}<d_{2}$.

Theorem 4.1. Suppose that (2.1), (2.2) and (3.1), (3.2) hold. If there exists an $H_{1} \in D\left(a_{i}, b_{i}\right)$ and $H_{2} \in D\left(c_{i}, d_{i}\right)$ such that either

$$
\sum_{n=a_{i}}^{b_{i}-1}\left[H_{1}^{2}(n+1)\left(p_{1}(n)+P_{\alpha}(n)\right) \frac{\tau(n)-\tau\left(a_{i}\right)}{n+1-\tau\left(a_{i}\right)}-\left(\Delta H_{1}(n)\right)^{2} k(n)\right] \geq 0
$$

or

$$
\sum_{n=c_{i}}^{d_{i}-1}\left[H_{2}^{2}(n+1)\left(p_{2}(n)+P_{\beta}(n)\right) \frac{\sigma\left(d_{i}\right)-\sigma(n)}{\sigma\left(d_{i}\right)-(n+1)}-\left(\Delta H_{2}(n)\right)^{2} k(n)\right] \geq 0
$$

for $i=1,2$, then $\left(E_{A, D}\right)$ is oscillatory. 